\title{
Study of Sex Differences In Systolic Blood Pressure and Exercise In Non- Athletic Adults
}

\author{
Sajja SK ${ }^{1}$, Deepthi $K^{2}$ \\ ${ }^{1}$ Dr Srikanth Sajja, Professor, Department of Physiology, ${ }^{2}$ Mrs Deepthi Kammili, Tutor, Department of Physiology. Both \\ affiliated with Dr PSIMS \& RF, Chinnavutapalli - 521286, Krishna, Andhra Pradesh. India
}

Address for correspondence: Dr. Srikanth Sajja, Email: minni_shp@yahoo.com

\begin{abstract}
Background \& Objectives: The aim of the present study was to study the sex differences in Systolic Blood Pressure (SBP) responses to Exercise in healthy, non- athletic adult's. SBP during exercise have been found to predict hypertension, coronary heart disease and cardiovascular disease (CVD). Materials \& Methods: One hundred normal, healthy, non-smoking subjects (50 Male \& 50 Female) of Dr. PSIMS\& RF are selected based on healthy life style, Body mass index, resting blood pressure $\&$ resting heart rate. Results: Age, BMI and pre-exercise heart rate showed no significant differences between genders. During the exercise test, males showed extremely significant \% rise in SBP \& HR than females. \% HR decline in 1st min and 3rd min has got extremely statistical significance between males and females. Systolic blood pressure recovery ratio shows extremely significant increase from 1 st $\min$ to 3 rd $\min$ in males. The \% HR decline in the 3 rd min showed extremely significant increase in males when compared to females. SBP responses during and after maximal exercise were estimated using percentage SBP rise (\%SBP rise) and SBP ratio in 3 minutes of recovery relative to 1 minute (SBPR2). Males showed a significantly higher \%SBP rise $(34.75 \pm 6.32 ; \mathrm{p}<0.001)$ and lower SBPR2 $(0.90 \pm 0.04 ; \mathrm{p}<0.001)$ in comparison to females. Conclusion: Exercise SBP testing provide an important clue to identify the subjects at risk for developing hypertension
\end{abstract}

Key words: Systolic Blood Pressure; Body Mass Index; Hypertension

\section{Introduction}

Researchers found that normotensive people who are at high risk of developing systemic hypertension had greater cardiovascular reactivity to physical stress. This normotensive's show an exaggerated blood pressure response to exercise test ${ }^{1}$. A rise in the SBP and a normal (or) low DBP is a normal response to isotonic exercise ${ }^{2}$. Studies have shown that persons with high-normal resting BP (or) unusually high BP response to exercise are prone to develop hypertension ${ }^{3}$. An elevated SBP at the $3 \mathrm{rd}$ minute of recovery and elevated exercise SBP response were also predictors of new onset hypertension ${ }^{4}$.

Increase in resting BP levels ${ }^{5}$ plays an important role in early detection \& identification of individuals with risk of developing Hypertension. Studies demonstrated that increase in BP that occurs during dynamic exercise is greater in Hypertensive's than in normotensive's ${ }^{6}$. In

Manuscript received: $23^{\text {rd }}$ Sept 2013

Reviewed: $26^{\text {th }}$ Sept 2013

Author Corrected: $29^{\text {th }}$ Oct 2013

Accepted for Publication: 30 ${ }^{\text {th }}$ Nov 2013 normal individuals SBP increase with dynamic exercise whereas DBP remains the same (or) falls slightly. In hypertensive individuals, rise in SBP\& DBP tends to be greater than in normal individuals ${ }^{7}$.

Considering the differences in blood pressure during exercise, systolic and diastolic pressure show different changes. With whole-body endurance activity, systolic blood pressure increases in direct proportion to increased exercise intensity. Increased systolic blood pressure results from the increased cardiac output that accompanies increasing rates of work. This helps to drive the blood quickly through the vasculature. Increased systolic pressure facilitates the delivery process. The vasoconstriction produced in the inactive tissues by the sympathetic nervous system and to some extent by the release of catecholamine's from the adrenal medulla is important for the maintenance of normal or increased blood pressure. Diastolic blood pressure changes little during endurance exercise, regardless of the intensity. When females and males are exposed to an acute bout of exercise, responses differ between the sexes in terms of 
strength, cardiovascular, respiratory responses and metabolic responses.

Exercise cardio acceleration results from release of parasympathetic inhibition at low exercise intensities and from both parasympathetic inhibition and sympathetic activation at moderate intensities ${ }^{1}$.

The sympathetic control of the microcirculation has been suggested to play an important role in the origin of Blood Pressure Variability ${ }^{8}$, 9 . In addition, blood pressure is regulated by baroreflex, a buffering mechanism that opposes increase or decrease of blood pressure by changes in heart rate, myocardial contractility, and peripheral resistance ${ }^{10}$. The arterial baroreflexes are reset to function at the prevailing arterial blood pressure of exercise.

The BP of exercise was probably the result of hemodynamic (Cardiac Output \& Total Peripheral Resistance) responses, which were regulated by two neural control systems, Central command and the Exercise pressor reflex.

\section{Materials \&methods}

One hundred normal, healthy, non-smoking subjects (students and staff) of Dr. PSIMS\& RF are selected based on healthy life style, body mass index measurement (computed as weight in kilograms divided by height in meters squared), resting blood pressure \& resting heart rate measurements.

Inclusion criteria : subjects who were non-athletic, nonobese (body mass index is <25) ,non-hypertensive (Systolic blood pressure should be in the range of 100-120 $\mathrm{mm}$ of $\mathrm{Hg}$, Diastolic blood pressure should be in the range of 70-80 mm of $\mathrm{Hg}$, non-smokers, non-alcoholics.

Exclusion criteria: subjects who were hypertensive (systolic blood pressure $>120$ and diastolic blood pressure $>90 \mathrm{~mm}$ of $\mathrm{Hg}$ ), athletes, obese (body mass index $>25$ ) and those taking medications that affect cardiovascular functions.

Subjects were informed of the experimental procedure and their consent was obtained before participation. The study was approved by the Institutional Ethics Committee. The resting blood pressure is measured using the Omron automatic blood pressure monitor with arm cuff (model HEM-711AC). The subject was instructed to rest for $10 \mathrm{~min}$ and the first reading was taken. After 15 minutes, the second reading was taken.

The average of the two values obtained was the resting blood pressure. The selected subjects underwent exercise test in the Clinical physiology lab. The exercise test was performed between 8:00 \& 11:00 AM in a well-ventilated room using a mechanically braked bicycle ergometer. Participants were instructed not to consume beverages containing alcohol, or coffee and not to eat a heavy meal or participate in any vigorous activity $24 \mathrm{hrs}$ before test.

On the test day subject's pre-exercise heart rate and blood pressure were measured twice. The subject was instructed to sit and rest on bicycle ergometer for $5 \mathrm{~min}$ and the first reading of blood pressure and heart rate reading was taken. After 10min, the second blood pressure and heart rate reading were taken using the electronic monitor.

The mean of the values was used as pre-exercise heart rate and blood pressure.

The exercise test protocol consisted of an initial $2 \mathrm{~min}$ warm up exercise at a work load of $180 \mathrm{~kg}-\mathrm{m} / \mathrm{min}(30$ watts workload). This was followed by an increase in workload of $120 \mathrm{~kg}-\mathrm{m} / \mathrm{min}$ (20watts) every minute till the subject complains of exhaustion.

During exercise the blood pressure and heart rate were measured for every $2 \mathrm{~min}$. The peak systolic blood pressure (peak SBP) and the peak heart rate (peak HR) are the highest values achieved during the test.

$\%$ rise of SBP $=$ Peak SBP - Pre exercise SBP $/$

Pre-exercise SBP x 100

$\%$ rise in HR $=$ Peak HR - Pre exercise HR/ Pre-exercise HR x 100

Post Exercise Protocol: Systolic BP was measured in $1^{\text {st }}$, 2nd $\& 3^{\text {rd }}$ min intervals after exercise. Post exercise SBP to peak exercise SBP ratio was calculated for $1^{\text {st }} \& 3^{\text {rd }}$ min intervals.

Post exercise SBP after $1 \mathrm{~min}$ of recovery to peak exercise ratio (SBPR1)

SBPR1 = Post SBP at 3rd min/ Peak SBP.

Post exercise SBP after $3 \mathrm{~min}$ of recovery to peak exercise ratio (SBPR2)

SBPR2 $=$ Post SBP at $3^{\text {rd }} \mathrm{min} /$ Post SBP at 1 st min. $\%$ decline of SBP during recovery was calculated as \% $\mathrm{SBP}$ decline $=$ Peak SBP - Post exercise SBP / Peak SBP x 100.

Post exercise HR was determined at 1 min of recovery and subsequently for every $2 \mathrm{~min}$ interval and discontinued as soon as SBP returns to pre exercise value. \% HR decline after exercise will be calculated as: $\% \mathrm{HR}$ decline = Peak HR - Post exercise HR / Peak HR x 100 


\section{Results}

Statistical analysis was done using Graphpad prism 6 software. Unpaired and paired t tests were used.

During the exercise test males showed extremely significant \% rise in SBP \& HR than females (\% SBP rise in Males - $34.75 \pm 6.32$; Female $-24.95 \pm 6.88, \%$ HR rise in Males $-103.7 \pm 12.98$; Female-74.97 \pm 18.61 , p value $<$ $0.0001)$. No correlation was observed between $\%$ rise in SBP and HR in males and females.

At post-exercise test males showed extremely significant higher values in \% SBP decline in 1 st and $3 \mathrm{rd} \min (\%$ SBP decline in $1 \mathrm{~min} \& 3 \mathrm{~min}$ in Males $-13.76 \pm 3.80 \&$ $21.44 \pm 3.72, \%$ SBP decline in $1 \mathrm{~min} \& 3 \mathrm{~min}$ in Females $-7.73 \pm 4.41 \& 12.26 \pm 4.37, \mathrm{p}<0.0001) . \%$ HR decline in $1 \mathrm{st}$ min and 3rd min has got extremely statistical significance between males and females (\% HR decline in $1 \mathrm{~min} \& 3 \mathrm{~min}$ in Males-22.53 $\pm 6.21 \& 32.06$ $\pm 7.53, \%$ HR decline in $1 \mathrm{~min} \& 3 \mathrm{~min}$ in Females -14.69 $\pm 4.99 \& 22.77 \pm 5.56, \mathrm{p}<0.0001)$. Males showed lower SBPR2 values than women (Male - SBPR2- $0.9 \pm 0.04$; Female- SBPR2- $0.95 \pm 0.03$ ) indicating that decline is more in 3rd min than females. Systolic blood pressure recovery ratio shows extremely significant increase from 1 st $\min$ to 3 rd $\min$ in males. Likewise, females also showed extremely significant increase in systolic blood pressure recovery ratio from 1 st $\min$ to 3 rd $\min$. The $\%$ HR decline in the 3rd min showed extremely significant increase in males when compared to females.

The results of our study showed gender differences in SBP responses, males exhibiting higher SBP response during and after exercise than females.

Table 1: Base line characteristics of the subjects

\begin{tabular}{|l|l|l|}
\hline CHARACTERISTICS & MALES & FEMALES \\
\hline AGE ( years ) & & \\
\hline BMI $\left(\mathbf{~ k g} / \mathbf{m}^{2}\right)$ & $23.08 \pm 3.82$ & $21.63 \pm 3.79$ \\
\hline SBP PRE (mmHg) & $121.7 \pm$ & $110.5 \pm$ \\
\hline DBP PRE (mmHg) & $79.3 \pm$ & $68.66 \pm$ \\
\hline HR PRE (bpm) & $79.04 \pm 7.76$ & $81.14 \pm 8.27$ \\
\hline
\end{tabular}

Age, BMI and pre-exercise heart rate showed no significant differences between genders. Pre-exercise diastolic blood pressure is found to be extremely significant between males and females (Male DBP $-80.19 \pm 5.26$; Female 72.22 \pm 7.43 , $\mathrm{p}$ value $<0.0001$ )

Table 2: Exercise test characteristics of the subjects

\begin{tabular}{|l|l|l|}
\hline Characteristics & MALES & FEMALES \\
\hline HR peak ( bpm) & $152.62 \pm 13.85$ & $151.98 \pm 12.80$ \\
\hline SBP peak ( mmHg) & $164.12 \pm 12.68$ & $146.52 \pm 11.71$ \\
\hline DBP peak (mmHg) & $86.5 \pm 9.09$ & $79.4 \pm 9.19$ \\
\hline \% SBP rise & $34.75 \pm 6.32$ & $24.45 \pm 6.88$ \\
\hline \% HR rise & $103.7 \pm 12.98$ & $74.97 \pm 18.61$ \\
\hline
\end{tabular}


Table 3: Post Exercise characteristics of the subjects

\begin{tabular}{|l|l|l|}
\hline & MALES & FEMALES \\
\hline SBP 1 min & $141.64 \pm 12.10$ & $127.6 \pm 11.00$ \\
\hline SBP 3 min & $128.78 \pm 9.20$ & $121.12 \pm 9.98$ \\
\hline HR 1 min & $117.7 \pm 12.29$ & $121.06 \pm 11.88$ \\
\hline HR 3 min & $102.4 \pm 11.92$ & $110.08 \pm 11.38$ \\
\hline \% SBP decline in 1 min & $13.76 \pm 3.80$ & $7.73 \pm 4.41$ \\
\hline \% SBP decline in 3 min & $21.44 \pm 3.72$ & $12.26 \pm 4.37$ \\
\hline \% HR decline in 1 min & $22.53 \pm 6.21$ & $14.69 \pm 4.99$ \\
\hline \% HR decline in 3 min & $32.06 \pm 7.53$ & $22.77 \pm 5.56$ \\
\hline SBPR & $0.78 \pm 0.04$ & $0.85 \pm 0.13$ \\
\hline SBPR 2 & $0.9 \pm 0.04$ & $0.95 \pm 0.03$ \\
\hline
\end{tabular}

\section{Discussion}

We studied about the assessment of Sex differences in SBP Responses to Exercise in Healthy Non-Athletic young adults. SBP and HR were measured during exercise at $2 \mathrm{~min}$ intervals, and post exercise at $2 \mathrm{~min}$ intervals, until SBP recovered to baseline. SBP responses during and after Bicycle Ergometer exercise (maximal) were assessed using \% SBP rise and SBP ratio in $3 \mathrm{~min}$ of recovery relative to $1 \mathrm{~min}$ (SBPR2).

In the present study, we calculated SBP response between males and females during maximal exercise using percentage SBP rise. Males experienced a significantly higher \% SBP rise $(34.75+6.32)$ and lower SBPR2 (0.90 $+0.04)$ in comparison to females. Age, Sex, education, Body weight, alcohol consumption, physical fitness, and medication have all been shown to affect the BP response to exercise independently and significantly ${ }^{\mathbf{1 1}}$.

Normotesive individuals with a hypertensive response to exercise have an increased risk at developing Hypertension in future ${ }^{12 ; 13}$. Dimpka $U$ et al ${ }^{14}$ studied about the assessment of Sex differences in SBP Responses to Exercise in Healthy Non-Athletic young adults. They measured the SBP and HR during exercise at $2 \mathrm{~min}$ intervals, and post exercise at 2 min intervals, until SBP recovered to baseline.

They observed that males experienced a significantly higher \% SBP rise $(39.82 \pm 9.52)$ and lower $\mathrm{SBPR}_{2}(0.92$ $\pm 0.06)$ in comparison to females. Also, they found a correlation between \% SBP rise and \% $\mathrm{HR}$ rise; $\mathrm{SBPR}_{2} \&$ $\mathrm{VO}_{2} \max ; \mathrm{SBPR}_{2}$ and \% $\mathrm{HR}$ decline in $1 \mathrm{~min}$ and $\mathrm{SBPR}_{2}$
\& $\%$ HR decline in 3 min were 27\%, 59\%, 32\%, 24\% in males and $4 \%, 34 \%, 25 \%, 23 \%$ in females. They concluded that males exhibited higher SBP response during and after exercise than females. The results of our study correlate with this study. A rise in SBP during exercise is mainly due to increase in cardiac output \& reflects the level of sympathetic and parasympathetic drive ${ }^{15}$.

Changes in the BP are usually mediated by the baroreflex mechanism via HR changes ${ }^{16}$.This baroreflex mediated response of $\mathrm{HR}$ to changes in arterial $\mathrm{BP}$ indicate the capacity of reflex cardiac autonomic modulation ${ }^{17}$. Fletcher GF, Balady GJ et al ${ }^{18}$ prescribed exercise standards for testing and training. They stated that in the early phases of exercise in the upright position, cardiac output is increased by an augmentation in stroke volume mediated through the use of frank-starling mechanism and heart rate.

The increase in Cardiac Output in the later phases of exercise is primarily due to an increase in Heart Rate. As exercise progresses, skeletal muscle blood flow is increased, oxygen extraction increases as much as 3 fold; total calculated peripheral resistance decrease and SBP, MAP \& PP usually increases. DBP may remain unchanged (or) decrease to a minimal degree. Normal values of SBP for men are directly related to Age.

Laitinen $\mathrm{T}$ et al ${ }^{19}$ evaluated the correlates of Baroreflex sensitivity (BRS) \& the role of sympathovagal balance in healthy subjects. They concluded that physiological 
factors age and gender have significant impact on BRS in healthy subjects. Impaired BRS has been suggested to play a role in the development of Hypertension. Reduced arterial compliance and increased sympathetic activity has been suggested to be responsible for the decreased BRS in Hypertension.

They also observed a positive correlation between BP \& Rhythmic Blood Pressure Variability (BPV) in healthy normotensive subjects and found that both systolic \& diastolic BPV were higher in women than in men. Salonen SK et al ${ }^{20}$ examined the relationship of exercise cardiac power (ECP), defined as a ratio of $\mathrm{VO}_{2}$ max with peak SBP during exercise, with the risk for stroke.

They observed that the association between ECP and increased risk of stroke was due to elevated after load and peripheral resistance. Exercise induced elevation of SBP has been found to be an independent predictor of stroke, hypertension, CHD and CVD death.

Singh JP 21 studied about the BP Response during treadmill testing as a risk factor for new onset hypertension - The Framingham Heart study. They observed that the mean resting, exercise and recovery SBP and DBP were higher in men than in women and they concluded that an exaggerated DBP response to exercise was predictive of risk for new-onset HTN in normotensive men and women. An elevated recovery SBP was predictive of HTN in men. Amon KW et al ${ }^{22}$ reviewed about the usefulness of post exercise response of SBP in the diagnosis of CAD.

They concluded that the normal decline in SBP during the recovery phase of treadmill exercise does not occur in some patients with CAD. They observed that the resting SBP were not significantly different between the two groups i.e., CAD group \& normal. But were significantly different during maximal exercise. Mean SBP recovery ratios at all $3 \mathrm{~min}$ of recovery were significantly different between the patients and the normal subjects. Scott AM et $\mathrm{al}^{23}$ reviewed about the delayed SBP recovery after graded exercise an independent correlate of angiographic coronary disease.

They concluded that adults showed delayed decline in SBP after exercise which was predictive of Coronary Artery Disease, even after taking into account the increase in BP during exercise. Gleim GW et al ${ }^{24}$ studied about the Gender differences in the systolic BP response to exercise. Men had significantly higher absolute SBP responses at $50 \%, 75 \%$ and $100 \%$ peak Heart rate on all modalities and they observed that this difference is absent when SBP is adjusted for Body Surface Area and is reduced when adjusted for body weights and reversed when SBP is adjusted for lean body mass. Stewart KJ et $\mathrm{al}^{25}$ studied about the blood pressure measures during exercise. They concluded that increase in Pulse Pressure is an indicator of Blood vessel stiffening. Drayer IM et $\mathrm{al}^{26}$ reviewed about BP as a determinant of cardiac left ventricular muscle mass. They concluded that the cardiac wall stress was the main determinant for the development of hypertrophy, and concluded that cardiac wall stress is in turn directly related to SBP. The Hemodynamic responses to leg cycling were typical for dynamic exercise of a large muscle mass.

This was marked by a widened pulse pressure, increase in cardiac output with HR \& Stoke Volume contributing to the effect and decrease in total peripheral resistance. Bezucha GR et al ${ }^{27}$ reported that increase in cardiac output mediated by Heart Rate may be a major determinant of the pressor response Shiomi $\mathrm{T}$ et al 28 highlighted the physiological responses and mechanical efficiency during different types of ergometric exercise, bilateral leg pedalling; (BLP); unilateral leg pedalling (ULP), bilateral Arm cranking (BAC) and unilateral arm cranking were due to disparities in the exerted muscle mass . Karen AM and Catherine MS ${ }^{29}$ reported that Men had greater SBP responses to all stressors than did women. Early rise of SBP during exercise adds prognostic information on cardio vascular mortality among healthy middle aged men with mildly elevated casual BP ${ }^{30}$. Males have a preponderance of sympathetic over vagal control of cardiac function compared with females ${ }^{(31-33)}$.

\section{Conclusion}

Blood pressure is determined by a complex interplay between cardiac output, which is related to left ventricular systolic function, and peripheral vascular resistance .The rate at which SBP decreases after exercise reflects a person's level of physical activity and fitness. Higher level of physical fitness was associated with more rapid decline of SBP and a greater decrease in SBP from peak exercise to the recovery reflects good aerobic capacity.

Persons with high normal resting blood pressure / an elevated response of $\mathrm{BP}$ to dynamic (or) isometric exercise were more prone to develop hypertension.

Exercise SBP testing may provide an additional tool to identify subjects at risk for developing hypertension in future.

Acknowledgements: We acknowledge all the subjects who have participated in this study and all the staff of the Department of Physiology for their cooperation.

Conflicts of interest: None declared

Source of funding: Nil

Available online at: $\underline{\text { www.ijmrr.in }}$

237| P a g e 


\section{References}

1. Wilson MF, Sung BH, Pincomb GA, Lovallo WR; Exaggerated blood pressure response to exercise in men at risk for systemic Hypertension. Am J Cardiol; 1990; 66:731-6.

2. Chaney RH, Eyman RK; BP at rest and during maximal dynamic \& isometric exercise as predictors of systemic Hypertension. Am J Cardiol; 1983; 62: 1058-61.

3. Singh JP, Larson MG, Manolio TA, O'Donnell CJ, Lauer M, Evans JC, Levy D; BP Response during treadmill testing as a risk factor for new onset Hypertension. Circulation; 1999; 99: 1831-6.

4. Lauer MS, Pashkow FJ, Harvey SA, Marwick TH, Thomas JD; Impaired chronotropic response to exercise stress testing as a predictor of mortality. JAMA 1999 , $281 ; 524-9$

5. Lane D, Beavers DG, Lip GYH; Ethnic differences in BP \& the prevalence of Hypertension in England. J Hum. Hypertens; 2002; 16:267-73.

6. Fagerd RH; Effects of exercise, diet, and their combination on BP.J Hum Hypertens ; 2005 Dec ; 19 :Suppl. 3 S20-4.

7. Dlin RA, Hanre N, Silver barg DS, Bar O; Follow up of normotensive men with exaggerated $\mathrm{BP}$ response to exercise. Am Heart Journal; 1983; 106:316-20.

8. Bernardi, L., D. Hayoz, R. Wenzel, C. Passino, A. Calciati, R.Weber, and G. Noll; Synchronous and baroreceptor-sensitive oscillations in skin microcirculation: evidence for central autonomic control. Am. J. Physiol.1997; 273 (Heart Circ. Physiol. 42):H1867-H1878.

9. Lossius K, Eriksen M and Walloe L; Fluctuations in blood flow to acral skin in humans: connection with heart rate and blood pressure variability. J. Physiol. (Lond.)1993; 460: 641-655.

10. Guyton AC and Hall JE; Nervous regulation of the circulation and rapid control of arterial pressure. In: Textbook of Medical Physiology (9th ed.), Philadelphia, PA: Saunders, 1994, 209-220

11. Criqui MH, Haskell WL, Heiss G, Tryoler HA, Green P, Rubenstein CJ; Predictors of systolic blood pressure response to treadmill exercise: the Lipid Research Clinics Program Prevalence Study. Circulation 1983; 68: 225-33.
12. Chaney RH; Eyman RK; BP at rest and during maximal dynamic \& isometric exercise as predictors of systemic Hypertension. Am J Cardiol; 1983: 62: 1058-61.

13. Jackson AS, Squires WG, Grimes G, Bread EF; Prediction of future resting hypertension from exercise blood pressure. J Cardiopul Rehab 1983; 3:263-8.

14. Dimpka U, Ugwu A, Oshi D; Assessment of sex differences in systolic blood pressure responses to exercise in healthy, non-athletic young adults. Journal of Exercise Physiology online. April 2008; Vol 11 (2), 1825.

15 Laukkanen JA, Sudhir K, Salonen R, Timo AL, Rauramaa R, Salonen JT ; Systolic blood pressure during recovery from exercise and the risk of acute myocardial infarction in middle aged men. Hypertension 2004; $44: 820-825$

16. FitPro TC; Blood pressure and exercise. American Fitness Professionals and Associates: 1998; retrieved on Nov 28, 2006 from http;www.afpafitness.com/articl/article-and-newsletter/.

17. Huikuri H V, Pikkujamsa SM , Airaksinen KEJ , Ikaheimo MJ , Rantala AO, Kauma H , Lilja M , and Kesaniemi A ; Sex-related differences in autonomic modulation of heart rate in middle-aged subjects. Circulation1996; 94: 122-125.

18. Fletcher GF, Balady GJ, Amsterdam EA, Chaitman B , Eckel R, Fleg J , Froelicher VF , Leon AS , Piña IL , Rodney R , Simons-Morton DA , Williams MA and Bazzarre $\mathrm{T}$; Exercise standards for testing and Training. A statement for health care Professionals from the American Heart Association. Circulation; 1992; 86:3404.

19. Laitinen T , Hartikainen J , Vanninen E , Niskanen L, Geelen G and Lansimies ; Age and gender dependency of baroreflex sensitivity in healthy subjects. J. Appl. Physiol.1998; 84(2): 576-583.

20. Salonen K, Laukkanen JA, Niskanen L, Rauramaa R , Tuomainen TP , Sivenius J and Salonen JT; Cardiac power during exercise and the risk of stroke in Men. Stroke 2005; 36:820-824.

21. Singh JP, Larson MG, Manolio TA, O`Donnell CJ, Lauer M, Evans JC, Levy D; Blood pressure response during treadmill testing as a risk factor for new-onset hypertension- The Framingham Heart Study. Circulation.1999; 99: 1831-1836. 
22. Amon $\mathrm{KW}$, Richards $\mathrm{KL}$ and Crawford $\mathrm{MH}$; Usefulness of the post exercise response of systolic blood pressure in the diagnosis of coronary artery disease. Circulation; 1984; 70:951-956.

23. Scott AM, Thomas HM, Fredric JP, Michael SL; Delayed systolic blood pressure recovery after graded exercise; An independent correlate of angiographic coronary disease. J. Am. Coll. Cardiol. 1999; 34:754759 .

24. Gleim GW, Stachenfeld NS, Coplan NL, Nicholas JA; Gender differences in the systolic blood pressure response to exercise. Am Heart J 1991; 121:524-530.

25. Stewart KJ , Anita CB, Katherine LT, Jamie R, De Regis; BP measures during exercise can indicate unhealthy hearts. Science Daily Sept18, 2001; http://www.jhbmc.jhu.edu/cardiology/rehab/hh-timonium.

26. Drayer IM, Weber MA, DeYoung JL; BP as a determinant of cardiac left ventricular muscle mass. Arch Int Med; 1983: 143:90-92.

27. Bezucha GR, Lenser MC, Hanson PG , Nagle FJ; Comparison of hemodynamic responses to static and dynamic exercise. J. Appl. Physiol.: Respirat. Environ. Exercise Physiol.1982, 53(6): 1589-1593
28. Shiomi T, Maruyama H, Saito A, Umemura M; Physiological responses and mechanical efficiency during different types of ergometric exercise. J Phys Ther Sci; $2000 ; 12 ; 67-73$.

29. Karen AM and Catherine M S; Influences of sex and age on cardiovascular responses during stress. Psychosomatic Medicine; 1988; 50; 46-56.

30. Mundal R, Kjeldsan SE, Sandvik L, Briknen G, Thaulow B ;Exercise BP predicts CV mortality in middle aged man. Hypertension; 1994; 24; 56-62.

31. Madden K, Savard GK; Effects of mental state on heart rate and blood pressure variability in men and women. ClinPhysiol 1995; 15:557-569.

32. Ramaekers D, Ector H, Aubert AE, Rubens A, Van de Werf $\mathrm{F}$; Heart rate variability and heart rate in healthy volunteers. Is the female autonomic nervous system cardioprotective. Eur Heart J 1998; 19: 1334-1341.

33. Yamasaki Y, Kodama M, Matsuhisa M; Diurnal heart rate variability in healthy subjects: effects of aging and sex difference. Am J Physiol 1996; 271: H303-H310.

\section{How to cite this article?}

Sajja SK, Deeepthi K. Study of Sex Differences In Systolic Blood Pressure And Exercise In Non- Athletic Adults. Int J Med Res Rev 2013; 1(5): 233-239. doi: 10.17511/ijmrr.2013.i5.04. 